\title{
MELAS, MIDD and Beyond: m.3243A>G MT-TL1 Mutation in Adult Patients
}

\author{
Bun Sheng1, Man Kei Fong1, Wing Kwan Ng1, Sammy Pak Lam Chen², Chloe Miu Mak² \\ ${ }^{1}$ Department of Medicine \& Geriatrics, Princess Margaret Hospital, Hong Kong, China \\ ${ }^{2}$ Department of Pathology, Princess Margaret Hospital, Hong Kong, China \\ Email: shengbun@hotmail.com
}

Received 4 July 2016; accepted 18 July 2016; published 21 July 2016

Copyright (C) 2016 by authors and Scientific Research Publishing Inc.

This work is licensed under the Creative Commons Attribution International License (CC BY).

http://creativecommons.org/licenses/by/4.0/

(c) (i) Open Access

\begin{abstract}
m.3243A>G MT-TL1 mutation is the most common mitochondrial DNA mutation that results in a wide spectrum of disorders in a maternally inherited pedigree. In adult patients, many present with symptoms and signs indistinguishable from acquired diseases and the correct diagnosis is often delayed after many years. Nevertheless, clues suggesting m.3243A $>$ G usually exist early in the disease course but are only realized late. These hints, from the evolution of symptoms and signs, family background, investigation results, or a combination of these, enable the physician to make the correct diagnosis early, which is important for appropriate treatment and better patient care. As with other inheritable diseases, genetic counselling should be offered regarding the disease management, inheritance mode, recurrence risk, usefulness and limitations of genetic testing and reproductive options.
\end{abstract}

\section{Keywords}

Mitochondrial Disease, MELAS, m.3243A>G

\section{Introduction}

Mitochondrial diseases are considered a category of "rare diseases", with a population prevalence of 1 in 5000 based on epidemiological studies [1] [2]. The $A>G$ transition of the mitochondrially encoded tRNA leucine 1 (UUA/G) gene at position 3243 (m.3243A>G, MT-TL1) is the most common mutation in human mitochondrial diseases [3]. This mutation accounts for most of the severe childhood neurological phenotype mitochondrial encephalomyopathy, lactic acidosis, and stroke-like episodes (MELAS), and maternally inherited diabetes and deafness (MIDD) in adults [4] [5]. These two discrete phenotypes are only part of the disease spectrum, many patients carrying this mutation do not fall into either group [6]. In a population epidemiological study, 1 in 400

How to cite this paper: Sheng, B., Fong, M.K., Ng, W.K., Chen, S.P.L. and Mak, C.M. (2016) MELAS, MIDD and Beyond: m.3243A>G MT-TL1 Mutation in Adult Patients. International Journal of Clinical Medicine, 7, 487-495.

http://dx.doi.org/10.4236/ijcm.2016.77054 
carried this m.3243A $>\mathrm{G}$ and most of them had deafness, which could actually be regarded as having mild disease [7]. In addition, these at risk persons could develop a full blown presentation in a later age, and their phenotype could also evolve. In this article, we share our experience in the clinical management of adult patients with this m.3243A $>\mathrm{G}$ mutation through a descriptive analysis to highlight the heterogeneity and diversity of the disease nature, and the treatment related issues.

\section{Our Patients}

\subsection{Case 1. Diabetic Patient with Acute Stroke Presentation, with MRI Showing MELAS but Not Infarction}

Mr A had a normal early development and childhood. His story started with progressive hearing loss since age 20. At 46 he was diagnosed diabetes mellitus (DM) in a health check, and he received metformin. Five years later, at 51, he had acute aphasia, and mild weakness over right side. Brain MRI revealed restricted diffusion over left temporal and parietal cortical ribbons that spared the subcortical white matters (Figure 1(A) and Figure $1(B)$ ). This radiological finger print, together with his background comorbidity of sensorineural deafness and DM in an underweighted man with BMI 17, prompted the suspicion of MELAS with acute stroke-like episode. We treated him with intravenous infusion of L-arginine followed by oral maintenance. His serum lactate was elevated at 3.0 (ref. 0.5 - 2.2) mmol/L, and an elevated lactate peak was present in the MR spectroscopy

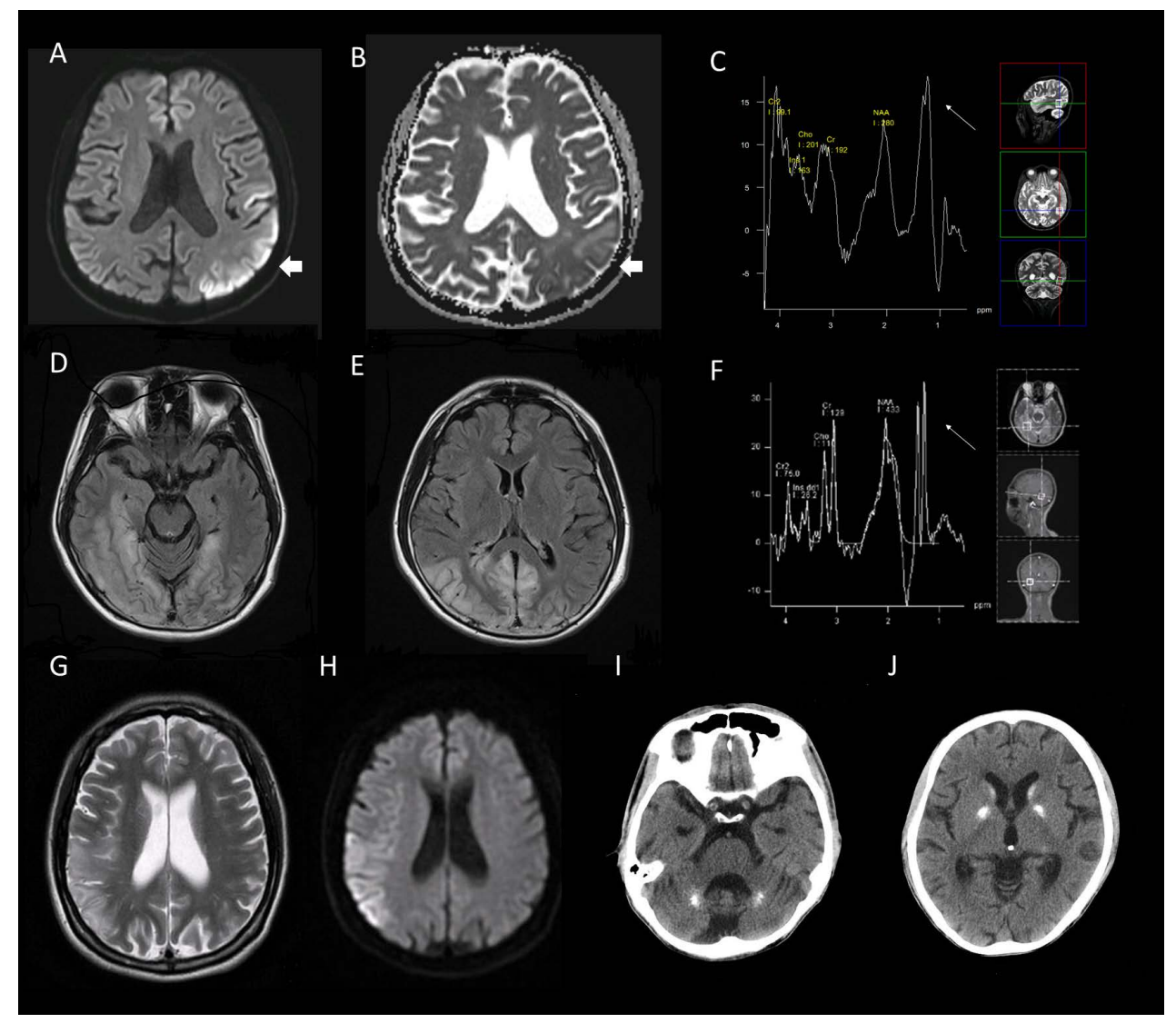

Figure 1. MRI of Mr A (case 1, A-C) showed restricted diffusion over left parietal and occpital cortex (arrow, A), without significant reduction of ADC map (arrow, B); MRS revealed a high lactate double peak at $1.33 \mathrm{ppm}$ (small arrow, C). MRI FLAIR of Mr C (case 3, D-F) showed gyral edema over bilateral occipital and temporal region, the edema was restricted to subcortical white matter (D \& E). MRS showed a high lactate double peak at 1.33 ppm (small arrow, F). G \& H: MRI of Miss E (case 5) during her second stroke-like episode showed hyperintense T2 lesion at right parietal cortex (G) with restricted diffusion (H). I \& J: Brain CT of Mr G (case 7) found cerebral and cerebellar atrophy with bilateral basal ganglia calcifications. 
(Figure 1(C)). The molecular genetics confirmed m.3243A>G, the commonest mutation for MELAS. Mr A had residual aphasia and cognitive impairment after the stroke-like episode, but remained ambulatory unaided and outdoor going. Metformin was switched to acarbose for his DM. He later developed simple partial seizure which was well controlled with lamotrigine. It has been two years since his stroke-like episode and he remained stable. The family history was also tale telling, with one of his six siblings died at young age from an unknown brain disease, and his mother, who was deceased many years ago, had long standing deafness.

\subsection{Case 2. Recurrent Syncope for More Than 10 Years-Time Will Tell}

Mr B was a chronic smoker. He had sensorineural deafness since his 30 s, chronic hepatitis B and DM. Started from $40 \mathrm{~s}$ he developed recurrent syncope with or without injury, and had repeated hospitalization up to four or five times a year for this problem. These syncope episodes were usually brief, with or without prodrome, exclusively at daytime, but lack of typical syndromic pattern for vasovagal syncope. He underwent very comprehensive diagnostic evaluation for a possible cardiac arrhythmia: multiple holter monitor, echocardiogram, treadmill, diagnostic coronary angiogram, cardiac electrophysiology study and finally implantable loop recorder, all were uneventful. He had been given empirical sodium valproate for suspected seizure related syncope without success, his electroencephalogram was normal. Having followed up his syncope problem for almost 10 years, he was found to have cardiomegaly and symptoms of heart failure. The repeated echocardiogram found dilated cardiomyopathy with poor ejection fraction of $24 \%$ only. That probably did not explain his long standing recurrent syncope. Further investigation noticed an abnormal autonomic function test with impairment mainly in sudomotor and cardiovagal pathway, and an inadequate cortisol response from the synacthen test. This was the time, at the age of 57, a genetic test for common mitochondrial mutations was requested, which confirmed m.3243A> G. The "MELAS" phenotype of Mr B consisted of sensorineural deafness, DM, adrenal insufficiency, autonomic neuropathy and cardiomyopathy. The reason for his recurrent syncope was not completely clear, but believed to be a combination of autonomic neuropathy and adrenal insufficiency in the early days, and was aggravated by cardiomyopathy in recent years.

\subsection{Case 3. A Fatal Stroke-Like Episode in a Young Man}

Mr C, a 29-year-old man, presented with acute left sided homonymous hemianopia, which rapidly progressed to total cortical blindness a day later. MRI brain showed bilateral parietal and occipital gyriform swelling (Figures 1(D)-(F)). Blood test found diabetes mellitus, a high random glucose $18 \mathrm{mmol} / \mathrm{L}$ and an elevated HbA1C 7; his serum lactate was $4.8 \mathrm{mmol} / \mathrm{L}$. His only significant past medical condition was pulmonary tuberculosis which he had a complete recovery five years ago; he did not have any symptoms of mitochondrial disease before. The clue for a possible mitochondrial disease in his family was traced to his mother, who had long standing deafness and died at the age of $50 \mathrm{~s}$ from a suspected cardiac condition. This unfortunate young man deteriorated rapidly despite intravenous L-arginine infusion, developed status epilepticus and deceased. The molecular genetics study identified m.3243 A>G. This is MELAS presented with a fatal stroke-like episode.

\subsection{Case 4. Multiple Somatic Complaints Did Not Equate Psychogenic}

Ms D was known to carry the m.3243A $>$ G mutation having her son diagnosed MELAS at childhood. She started to feel unwell in her $40 \mathrm{~s}$. She had deafness and dyspepsia symptoms with pain and distension, for which she paid frequent visits to general outpatient, almost weekly. The family clinic physician marked down "multiple complaints!!” in the notes and believed a strong psychological component underlie her symptoms. At age 55 she developed first stroke-like episode with left sided hemi-neglect, ideomotor apraxia and weakness. CT brain revealed right parietal hypodensity. She was treated with L-arginine infusion. This attack was complicated by myoclonic seizure which was controlled with levetiracetam. She recovered from this episode with only mild residual cognitive impairment. One year later she had another stroke-like episode over left parietal region with aphasia. Her third stroke-like episode was stormy; she had a new lesion over left temporal region, and a prolonged refractory status epilepticus which was eventually controlled with phenobarbitone, lacosamide and levetiracetam. Her cognitive impairment worsened after these three stroke-like episodes but she remained ambulatory and independent in her basic self-care. She still complained of abdominal discomfort, but that appeared to be a lesser concern now. We believed it was mitochondrial enteropathy. With a correct diagnosis, things make perfect sense. 


\subsection{Case 5. Birth Asphyxia or MELAS to Be Blamed for Developmental Delay?}

Miss E was believed to have developmental delay and learning disability from birth asphyxia. She presented initially at age 33 with gradual onset of hearing loss and aphasia. Together with these she also developed simple partial seizure with twitching of right-sided face and limbs. MRI brain revealed left temporal and parietal gyraloedema. Serum lactate was high at $4.5 \mathrm{mmol} / \mathrm{L}$. A muscle biopsy was performed but there was no diagnostic feature for mitochondrial cytopathy. She improved and was able to be weaned off from anticonvulsant. Three years later, she suffered another similar attack, presented with involuntary limb movements. The second MRI brain showed new right temporal and parietal gyraloedema (Figure 1(G) and Figure 1(H)). The molecular genetics again confirmed m.3243A>G. Subsequently, she developed DM, and became insulin dependent shortly afterwards. Migraine was another frequent complaint. These evolving signs and symptoms down the years summarized a typical MELAS phenotype. MELAS should also be accountable for the learning disability in this lady since there was obvious gradual cognitive decline over time.

\subsection{Case 6. Severe Insulin Resistance, Liver Derangement, and Psychosis}

Miss F came from a broken family. Her mother died of carcinoma of pancreas, and she had a very poor relationship with her father and the step mother. She left the family since teenage, and addicted to multiple substance abuse. She noticed progressive hearing loss and tinnitus when she reached early $20 \mathrm{~s}$, and she wore hearing aid few years later. At 25 she had psychotic symptoms of delusion and auditory hallucination, which was attributed to her substance abuse, and antipsychotics were given. Three years later she had a period of intermittent abdominal cramps, and blood test found persistent elevated liver enzymes ranging from 120 to 300 (ref. < 35) U/L. The usual medical work up for hepatitis was uneventful, and the liver ultrasound only showed fatty changes. A few months later, blood test found hyperglycemia, her HbA1C level was elevated to 15 . Gliclazide and metformin were added for the newly diagnosed DM. Miss F was short and slim, her BMI was 17 only; she did not resemble a typical type II DM patient. Within the same year, she started to have muscle weakness and unsteadiness. The electromyography revealed myopathic changes, her CK level was normal, but lactate was elevated at $4.3 \mathrm{mmol} / \mathrm{L}$. The muscle biopsy confirmed mitochondrial myopathy, and the genetic study was positive for $\mathrm{m} .3243 \mathrm{~A}>\mathrm{G}$. The most striking management issue in this lady was the high insulin requirement that signified the degree of insulin resistance. Insulin was started about one year after her diagnosis of DM because of the persistent high HbA1C ranging from 9 - 12. Within one year, the daily insulin dosage raised to 132 units, which was equivalent to 3.7 unit/kg/day. Together with pioglitazone and acarbose, her latest HbA1C level was only marginally improved to 9.9. The myopathy also deteriorated, she could barely walk for short distance with a frame three years after the initial presentation of weakness. Her cardiac function was normal. The liver enzyme (ALT) still fluctuated between 100 and $500 \mathrm{IU} / \mathrm{L}$, it might well be a form of mitochondrial hepatopathy, though not commonly reported in MELAS. We do not undercount substance abuse in her psychosis because of their well-known association, though MELAS with schizophrenia-like illness has also been reported.

\subsection{Case 7. MELAS with Mild Lactate Elevation}

Mr G had hearing loss since teenage. He was diagnosed to have DM in his early $30 \mathrm{~s}$. His blood pressure was all along low with SBP ranging 80 - $90 \mathrm{mmHg}$ only. The echocardiogram revealed normal cardiac function, and the cortisol level was adequate. He developed frequent abdominal pain at around 40 , and was admitted many times with repeated vomiting, dehydration, and intestinal pseudo-obstruction. During these admissions his glycemic control fluctuated and he had acute kidney injury with raised creatinine up to $300 \mu \mathrm{mol} / \mathrm{L}$. In one of these admissions he developed hypoglycemia and persistent metabolic acidosis, with a mildly elevated serum lactate at $2.7 \mathrm{mmol} / \mathrm{L}$. Both of his hypoglycemia and high lactate level were corrected with glucose and fluid replacement, but his metabolic acidosis persisted with $\mathrm{pH}$ 7.18, HCO3 $14 \mathrm{mmol} / \mathrm{L}$ and $\mathrm{BE}-12.8 \mathrm{mmol} / \mathrm{L}$. His urine $\mathrm{pH}$ was 6.2, the anion gap was normal, and his chloride level was $114(101-109) \mathrm{mmol} / \mathrm{L}$. This biochemical profile suggested renal tubular acidosis. His brain CT, at a young age of 45 , had already showed marked cerebral and cerebellar atrophy with bilateral basal ganglia calcifications (Figure 1(I) and Figure 1(J)). The molecular genetics confirmed m. 3243A>G. Mr G has this 'MELAS' phenotype of DM, deafness, enteropathy, and renal tubular disease, and possibly autonomic neuropathy to account for his hypotension. MELAS, but not lactic acidosis, is the lesson to learn. 


\section{Diagnosis}

The above cases demonstrated the diversity of disease presentations in this m.3243A $>$ G mutation. Patients could started with mild symptoms at childhood and gradually accumulate new symptoms, or present acutely at older age. The diagnosis may not be suspected as many of the symptoms are also commonly seen in acquired diseases. The long latency in reaching the correct diagnosis, often in term of decades, are not uncommon. It is the combination of symptoms \& signs from multiple organ system, together with a compatible family history of maternal inheritance, that raise the index of suspicion. Deafness is a common symptom at early phase, attentive to this “deafness plus syndrome” provides clue to m.3243A>G.

Good biomarkers for mitochondrial disease is lacking. An elevated blood lactate helps, the yield increases with post-prandial blood sampling, but some patients still have normal lactate level. Serum fibroblast growth factor 21 (FGF-21) has recently be developed as a biomarker for mitochondrial disease with muscle weakness, to distinguish mitochondrial myopathy from other neuromuscular conditions [8]. Its use is only limited to patients with myopathy. It correlates poorly with disease severity and it is not a prognostic marker [9]. Molecular genetics study, from blood, urine or tissue samples, confirms the diagnosis. Blood samples usually have lower level of heteroplasmy compared to urinary epithelial cell and muscle tissue, and its level also decreases with age [10]. Therefore, in older adults it is preferable to use urine or tissue samples for the genetic study.

\section{Organ Specific Manifestations}

\subsection{Stroke-Like Episodes}

The most important thing is to recognize the characteristic radiological change, which is best appreciated from MRI. Typically, the acute lesion locates at parietal and occipital region, involve predominantly cortical and subcortical white matter, and spares the deep white matter [11] [12]. There is no clot occluding the vessels despite having large cortical lesions, and the lesion distributions do not conform to vascular territory. The acute lesion shows restricted diffusion (appears bright on DWI), but without significant reduction in apparent diffusion coefficient (ADC) maps [13]. This suggests that most of the swelling seen on MRI is from vasogenic rather than cytotoxic oedema. Migration of lesions may be appreciated with serial monitoring. MR spectroscopy usually demonstrates a high lactate peak in acute lesions, and, with an acute infarction excluded from the lack of darkening on ADC map, points to MELAS [14]. This elevated lactate, if being detected in brain regions spared from acute lesion, also supports the diagnosis.

\subsection{Epilepsy}

The seizure in MELAS patients is frequently associated with stroke-like episode, just as described in our patients, and then recurs intermittently. Simple motor seizure is the most frequent type. During stroke-like events the seizure could be prolonged, evolving into convulsive or non-convulsive status epilepticus, or epilepsia partialis continua [15].

\subsection{Muscle and Heart}

Weakness and poor exercise tolerance are prevalent among m.3243A>G carriers. Cardiomyopathy, both dilated and hypertrophic, have been reported [16]. Arrhythmia, such as Wolff-Parkinson-White syndrome, could be another cardiac manifestation [17].

\subsection{Diabetes Mellitus}

MIDD is a recognized entity and affects about 1\% of DM patients [18]. Phenotypically they are young onset DM with a low BMI [19]. Many of them require insulin therapy two years after their diagnosis. Recent studies suggested insulin resistance and relative insulin deficiency is the main reason for MIDD, although the insulin secretion from pancreatic $\beta$-cell is dependent on mitochondrial function [20] [21].

\subsection{Hepatopathy and Enteropathy}

MELAS patients frequently complain of anorexia and poor weight gain. All sorts of gastrointestinal symptoms, 
from dyspepsia, vomiting, abdominal cramp, constipation, diarrhoea, to intestinal pseudo-obstruction, have all been described [22] [23]. Hepatopathy, usually in childhood, has been reported in other mitochondrial diseases, whether m.3243A>G mutation causes hepatopathy is unclear. Recurrent pancreatitis is another recognized visceral organ presentation.

\subsection{Nephropathy}

Proteinuria and impaired kidney function could be a feature in this spectrum. Focal segmental glomerulosclerosis, proximal tubular defects and tubulointerstitial nephropathy have been documented [24] [25].

\section{Management}

\subsection{Acute Treatment in Stroke-Like Episodes}

Arginine supplement is the only evidence-based specific treatment for MELAS in acute stroke-like episode for a quick recovery, and to prevent subsequent similar events [26]. MELAS patients in acute stroke-like episode have arginine deficiency, that is believed to further compromise the nitric oxide (NO) dependent endothelial function and vasodilatation [27]. In their early studies, Koga \& Co found replacement of arginine through intravenous infusion improved neurological symptoms rapidly within 24 hours, and that was accompanied by improved perfusion in SPECT [28] [29]. Our own experience in intravenous L-arginine is not encouraging, and we believe that discrepancy is because of treatment delay, since the study patients were treated within one hour of symptoms onset, but none of our patient was treated within 24 hours. In order to narrow the gap, a letter or remark in the electronic record system is necessary to alert the in-charge physician for urgent prescription. The regime is L-arginine $0.5 \mathrm{~g} / \mathrm{kg}$ in normal saline making up a $10 \%$ solution, infused over 15 minutes, followed by oral L-arginine $0.3 \mathrm{~g} / \mathrm{kg} /$ day in three or four divided doses.

\subsection{Management of Co-Morbidities}

Drug treatments are required for the management of multiple co-morbidities in these patients. In choosing the appropriate agents caution should be taken to avoid those drugs with potential harmful effect on mitochondrial function or oxidative phosphorylation. Valproate, with reports documenting hepatic failure and worsening of MELAS, is the most important agent to avoid [30]. Phenobarbitone and phenytoin should also be used with caution. Metformin is another agent to avoid in the fear of lactic acidosis although there is little evidence in this. Thiazolidinediones impair cell respiration through their effect on complex I, they should also be used with caution [31]. Other potentially harmful agents include statins, fibrate, $\beta$-blocker, amiodarone and neuroleptics such as chlorpromazine, haloperidol, risperidone and quetiapine.

\subsection{Exercise}

It is well known that exercise improves mitochondrial function. Exercise has become another evidence-based treatment in mitochondrial myopathy. Consistently, endurance exercise improves oxidative capacity and quality of life, while resistance exercise increases muscle strength, and may decrease the \% heteroplasmy in the muscle [32]-[35]. The latter is more prominent in single large mitochondrial DNA deletions, whether point mutations like m.3243A $>\mathrm{G}$ could have the similar benefit is unclear. This gain is thought to be derived from a "genetic drift" phenomenon, where the satellite cells within the muscle could be activated through resistance exercise and fusing their wild type mitochondrial DNA to the muscle cell and dilute the heteroplamy [36]. Therefore, every mitochondrial disease patient should has an exercise program starts at a lower intensity level according his tolerance, and builds up gradually. This should be a long term strategy.

\subsection{Genetic Counselling and Prenatal Diagnosis}

Genetic counselling is important for the patient and family to understand the disease, mode of inheritance, possible future outcome, and for reproductive planning [37]. With improving technologies in mitochondrial DNA heteroplasmy analyses, it has been advocated for prenatal testing for mitochondrial disease in some countries [38]. Unfortunately, in m.3243A>G mutation, the \% heteroplasmy correlates poorly with phenotype and prognosis, this would significantly limit the interpretation of prenatal testing result, especially in those with interme- 
diate heteroplasmy level [39]. Besides, affected mother would invariably pass on the mutation to all of her offsprings. The latest mitochondrial donation technology could provide women with another option to stop passing the mutation to their offspring.

\section{Conclusion}

m.3243A $>\mathrm{G}$ is a true big mimicker, the full picture of an affected patient would only be understood through long term follow up. With a high index of suspicion, diagnosis could be reached earlier and appropriate treatment administered. Genetic counselling, reproductive planning and prenatal diagnosis would help to prevent the disease in the family.

\section{Conflicts of Interest}

None.

\section{References}

[1] Skladal, D., Halliday, J. and Thorburn, D.R. (2003) Minimum Birth Prevalence of Mitochondrial Respiratory Chain Disorders in Children. Brain, 126, 1905-1912. http://dx.doi.org/10.1093/brain/awg170

[2] Gorman, G.S., Schaefer, A.M., Ng, Y., Gomez, N., Blakely, E.L., Alston, C.L., et al. (2015) Prevalence of Nuclear and Mitochondrial DNA Mutations Related to Adult Mitochondrial Disease. Annals of Neurology, 77, 753-759. http://dx.doi.org/10.1002/ana.24362

[3] Cao, Y., Ma, Y., Zhang, Y., Li, Y., Fang, F., Wang, S., et al. (2010) Detection of Eight Frequently Encountered Point Mutations in Mitochondria in Chinese Patients Suggestive of Mitochondrial Encephalomyopathies. Mitochondrion, 10, 330-334. http://dx.doi.org/10.1016/j.mito.2010.01.008

[4] Sproule, D.M. and Kaufmann, P. (2008) Mitochondrial Encephalopathy, Lactic Acidosis, and Strokelike Episodes: Basic Concepts, Clinical Phenotype, and Therapeutic Management of MELAS Syndrome. Annals of the New York Academy of Sciences, 1142, 133-158. http://dx.doi.org/10.1196/annals.1444.011

[5] Kadowaki, T., Kadowaki, H., Mori, Y., Tobe, K., Sakuta, R., Suzuki, Y., et al. (1994) A Subtype of Diabetes Mellitus Associated with a Mutation of Mitochondrial DNA. New England Journal of Medicine, 330, 962-968. http://dx.doi.org/10.1056/NEJM199404073301403

[6] Nesbitt, V., Pitceathly, R.D., Turnbull, D.M., Taylor, R.W., Sweeney, M.G., Mudanohwo, E.E., et al. (2013) The UK MRC Mitochondrial Disease Patient Cohort Study: Clinical Phenotypes Associated with the m.3243A>G MutationImplications for Diagnosis and Management. Journal of Neurology, Neurosurgery and Psychiatry, 84, 936-938. http://dx.doi.org/10.1136/jnnp-2012-303528

[7] Manwaring, N., Jones, M.M., Wang, J.J., Rochtchina, E., Howard, C., Mitchell, P. and Sue, C.M. (2007) Population Prevalence of the MELAS A3243G Mutation. Mitochondrion, 7, 230-233. http://dx.doi.org/10.1016/j.mito.2006.12.004

[8] Suomalainen, A., Elo, J.M., Pietilainen, K.H., Hakonen, A.H., Sevastianova, K., Korpela, M., et al. (2011) FGF-21 as a Biomarker for Muscle-Manifesting Mitochondrial Respiratory Chain Deficiencies: A Diagnostic Study. Lancet Neurology, 10, 806-818. http://dx.doi.org/10.1016/S1474-4422(11)70155-7

[9] Koene, S., de Laat, P., van Tienoven, D.H., Vriens, D., Brandt, A.M., Sweep, F.C., et al. (2014) Serum FGF21 Levels in Adult m.3243A>G Carriers: Clinical Implications. Neurology, 83, 125-133. http://dx.doi.org/10.1212/WNL.0000000000000578

[10] Shanske, S., Pancrudo, J., Kaufmann, P., Engelstad, K., Jhung, S., Lu, J., et al. (2004) Varying Loads of the Mitochondrial DNA A3243G Mutation in Different Tissues: Implications for Diagnosis. American Journal of Medical Genetics Part A, 130A, 134-137. http://dx.doi.org/10.1002/ajmg.a.30220

[11] Saneto, R.P., Friedman, S.D. and Shaw, D.W.W. (2008) Neuroimaging of Mitochondrial Disease. Mitochondrion, 8, 396-413. http://dx.doi.org/10.1016/j.mito.2008.05.003

[12] Abe, K., Yoshimura, H., Tanaka, H., Fujita, N., Hikita, T. and Sakoda, S. (2004) Comparison of Conventional and Diffusion-Weighted MRI and Proton MR Spectroscopy in Patients with Mitochondrial Encephalomyopathy, Lactic Acidosis, and Stroke-Like Events. Neuroradiology, 46, 113-117. http://dx.doi.org/10.1007/s00234-003-1138-2

[13] Oppenheim, C., Galanaud, D., Samson, Y., Sahel, M., Dormont, D., Wechsler, B. and Marsault, C. (2000) Can Diffusion Weighted Magnetic Resonance Imaging Help Differentiate Stroke from Stroke-Like Events in MELAS? Journal of Neurology, Neurosurgery and Psychiatry, 69, 248-250. http://dx.doi.org/10.1136/jnnp.69.2.248 
[14] Jose da Rocha, A., Tulio Braga, F., Carlos Martins Maia Jr., A., Jorge da Silva, C., Toyama, C., Pereira Pinto Gama, H., et al. (2008) Lactate Detection by MRS in Mitochondrial Encephalopathy: Optimization of Technical Parameters. Journal of Neuroimaging, 18, 1-8. http://dx.doi.org/10.1111/j.1552-6569.2007.00205.X

[15] Demarest, S.T., Whitehead, M.T., Turnacioglu, S., Pearl, P.L. and Gropman, A.L. (2014) Phenotypic Analysis of Epilepsy in the Mitochondrial Encephalomyopathy, Lactic Acidosis, and Strokelike Episodes-Associated Mitochondrial DNA A3243G Mutation. Journal of Child Neurology, 29, 1249-1256. http://dx.doi.org/10.1177/0883073814538511

[16] Vydt, T.C., de Coo, R.F., Soliman, O.I., Ten Cate, F.J., van Geuns, R.J., Vletter, W.B., et al. (2007) Cardiac Involvement in Adults with m.3243A > G MELAS Gene Mutation. American Journal of Cardiology, 99, 264-269. http://dx.doi.org/10.1016/j.amjcard.2006.07.089

[17] Sproule, D.M., Kaufmann, P., Engelstad, K., Starc, T.J., Hordof, A.J. and De Vivo, D.C. (2007) Wolff-ParkinsonWhite Syndrome in Patients with Melas. Archives of Neurology, 64, 1625-1627. http://dx.doi.org/10.1001/archneur.64.11.1625

[18] Ng, M.C., Yeung, V.T., Chow, C.C., Li, J.K., Smith, P.R., Mijovic, C.H., et al. (2000) Mitochondrial DNA A3243G Mutation in Patients with Early- or Late-Onset Type 2 Diabetes Mellitus in Hong Kong Chinese. Clinical Endocrinology, 52, 557-564. http://dx.doi.org/10.1046/j.1365-2265.2000.00989.x

[19] Murphy, R., Turnbull, D.M., Walker, M. and Hattersley, A.T. (2008) Clinical Features, Diagnosis and Management of Maternally Inherited Diabetes and Deafness (MIDD) Associated with the 3243A > G Mitochondrial Point Mutation. Diabetic Medicine, 25, 383-399. http://dx.doi.org/10.1111/j.1464-5491.2008.02359.x

[20] El-Hattab, A.W., Emrick, L.T., Hsu, J.W., Chanprasert, S., Jahoor, F., Scaglia, F. and Craigen, W.J. (2014) Glucose Metabolism Derangements in Adults with the MELAS m.3243A > G Mutation. Mitochondrion, 18, 63-69. http://dx.doi.org/10.1016/j.mito.2014.07.008

[21] Wiederkehr, A. and Wollheim, C.B. (2012) Mitochondrial Signals Drive Insulin Secretion in the Pancreatic Beta-Cell. Molecular and Cellular Endocrinology, 353, 128-137. http://dx.doi.org/10.1016/j.mce.2011.07.016

[22] Rahman, S. (2013) Gastrointestinal and Hepatic Manifestations of Mitochondrial Disorders. Journal of Inherited Metabolic Disease, 36, 659-673. http://dx.doi.org/10.1007/s10545-013-9614-2

[23] Chapman, T.P., Hadley, G., Fratter, C., Cullen, S.N., Bax, B.E., Bain, M.D., et al. (2014) Unexplained Gastrointestinal Symptoms: Think Mitochondrial Disease. Digestive and Liver Disease, 46, 1-8. http://dx.doi.org/10.1016/j.dld.2013.04.008

[24] Martin-Hernandez, E., Garcia-Silva, M.T., Vara, J., Campos, Y., Cabello, A., Muley, R., et al. (2005) Renal Pathology in Children with Mitochondrial Diseases. Pediatric Nephrology, 20, 1299-1305. http://dx.doi.org/10.1007/s00467-005-1948-z

[25] Emma, F., Montini, G., Salviati, L. and Dionisi-Vici, C. (2011) Renal Mitochondrial Cytopathies. International Journal of Nephrology, 2011, Article ID: 609213. http://dx.doi.org/10.4061/2011/609213

[26] Koga, Y., Povalko, N., Nishioka, J., Katayama, K., Kakimoto, N. and Matsuishi, T. (2010) MELAS and L-Arginine Therapy: Pathophysiology of Stroke-Like Episodes. Annals of the New York Academy of Sciences, 1201, 104-110. http://dx.doi.org/10.1111/j.1749-6632.2010.05624.x

[27] Koga, Y., Povalko, N., Nishioka, J., Katayama, K., Yatsuga, S. and Matsuishi, T. (2012) Molecular Pathology of MELAS and L-Arginine Effects. Biochimicaet Biophysica Acta, 1820, 608-614. http://dx.doi.org/10.1016/j.bbagen.2011.09.005

[28] Koga, Y.M.D.P., Ishibashi, M.M.D.P., Ueki, I.M., Yatsuga, S.M., Fukiyama, R.M., Akita, Y.M.D.P. and Matsuishi, T.M.D.P. (2002) Effects of L-Arginine on the Acute Phase of Strokes in Three Patients with MELAS. Neurology, 58, 827-828. http://dx.doi.org/10.1212/WNL.58.5.827

[29] Koga, Y.M.D.P., Akita, Y.M.D.P., Nishioka, J.M., Yatsuga, S.M., Povalko, N.M., Tanabe, Y.M.D.P., et al. (2005) L-Arginine Improves the Symptoms of Strokelike Episodes in MELAS. Neurology, 64, 710-712. http://dx.doi.org/10.1212/01.WNL.0000151976.60624.01

[30] Nanau, R.M. andNeuman, M.G. (2013) Adverse Drug Reactions Induced by Valproic Acid. Clinical Biochemistry, 46, 1323-1338. http://dx.doi.org/10.1016/j.clinbiochem.2013.06.012

[31] Finsterer, J. and Segall, L. (2010) Drugs Interfering with Mitochondrial Disorders. Drug and Chemical Toxicology, 33, 138-151. http://dx.doi.org/10.3109/01480540903207076

[32] Jeppesen, T.D., Schwartz, M., Olsen, D.B., Wibrand, F., Krag, T., Duno, M., et al. (2006) Aerobic Training Is Safe and Improves Exercise Capacity in Patients with Mitochondrial Myopathy. Brain, 129, 3402-3412. http://dx.doi.org/10.1093/brain/awl149

[33] Jeppesen, T.D., Duno, M., Schwartz, M., Krag, T., Rafiq, J., Wibrand, F. and Vissing, J. (2009) Short- and Long-Term Effects of Endurance Training in Patients with Mitochondrial Myopathy. European Journal of Neurology, 16, 13361339. http://dx.doi.org/10.1111/j.1468-1331.2009.02660.x 
[34] Bishop, D.J., Granata, C. and Eynon, N. (2014) Can We Optimise the Exercise Training Prescription to Maximise Improvements in Mitochondria Function and Content? Biochimicaet Biophysica Acta, 1840, 1266-1275. http://dx.doi.org/10.1016/j.bbagen.2013.10.012

[35] Tarnopolsky, M.A. (2014) Exercise as a Therapeutic Strategy for Primary Mitochondrial Cytopathies. Journal of Child Neurology, 29, 1225-1234. http://dx.doi.org/10.1177/0883073814538512

[36] Taivassalo, T., Fu, K., Johns, T., Arnold, D., Karpati, G. and Shoubridge, E.A. (1999) Gene Shifting: A Novel Therapy for Mitochondrial Myopathy. Human Molecular Genetics, 8, 1047-1052. http://dx.doi.org/10.1093/hmg/8.6.1047

[37] Vento, J.M. and Pappa, B. (2013) Genetic Counseling in Mitochondrial Disease. Neurotherapeutics, 10, $243-250$. http://dx.doi.org/10.1007/s13311-012-0173-2

[38] Nesbitt, V., Pitceathly, R.D., Turnbull, D.M., Taylor, R.W., Sweeney, M.G., Mudanohwo, E.E., et al. (2013) The UK MRC Mitochondrial Disease Patient Cohort Study: Clinical Phenotypes Associated with the m.3243A > G Mutation-Implications for Diagnosis and Management. Journal of Neurology, Neurosurgery and Psychiatry, 84, 936-938. http://dx.doi.org/10.1136/jnnp-2012-303528

[39] Poulton, J. and Marchington, D.R. (2000) Progress in Genetic Counselling and Prenatal Diagnosis of Maternally Inherited mtDNA Diseases. Neuromuscular Disorders, 10, 484-487. http://dx.doi.org/10.1016/S0960-8966(00)00130-9

\section{Submit or recommend next manuscript to SCIRP and we will provide best service for you:}

Accepting pre-submission inquiries through Email, Facebook, LinkedIn, Twitter, etc.

A wide selection of journals (inclusive of 9 subjects, more than 200 journals)

Providing 24-hour high-quality service

User-friendly online submission system

Fair and swift peer-review system

Efficient typesetting and proofreading procedure

Display of the result of downloads and visits, as well as the number of cited articles

Maximum dissemination of your research work

Submit your manuscript at: http://papersubmission.scirp.org/ 